\title{
Predictive Surrogate Markers of Allergy: A By-Product of Recombinant Allergens?
}

\author{
Claude André \\ Scientific and Medical Department, Stallergènes SA, Antony, France
}

For many years, allergy research has been trying to identify predictive markers of clinical reactivity in order to distinguish between simple biological sensitization and true clinical disease due to allergy. Evidence of true allergic disease is not always available and challenge tests are not sufficiently used. Another important issue is how to determine which patients are candidates for immunotherapy. As polysensitization is a fairly common phenomenon, which allergens should be used for immunotherapy? Can the short-term and long-term success of this treatment be predicted? What are the risk factors for progression of allergy towards a more severe form, such as progression from rhinitis to asthma? Is it possible to identify patients at high risk of serious anaphylactic reactions during immunotherapy?

Progress in biology, and especially the availability of very good-quality recombinant allergens, now provides a clearer understanding of allergic diseases. A particularly important contribution was provided by the study by Pastorello et al. [1] in the International Archives of Allergy Immunology, which described, in a large series of patients, the severity of allergic reactions according to the type of sensitizations in a context of pollen-food crossreactivity.

In the somewhat distant past, in vivo diagnostic tests, such as prick tests, or in vitro diagnostic tests, such as specific IgE assay, correlated the intensity of sensitization to the severity of clinical features. For example, in asthmatic children sensitized to house dust mites, allergy is confirmed when the wheal on prick tests is greater than or equal to $6 \mathrm{~mm}$, and allergy is probable when the wheal is greater than or equal to $5 \mathrm{~mm}$ [2]. However, skin prick tests cannot constitute a universal criterion due to variations in the biological potency of allergen extracts between various manufacturers, and sometimes even from the same manufacturer. The validity of skin tests in food allergy has also been demonstrated [3], particularly in terms of sensitivity and negative predictive value. According to Sampson and Ho [3], a specific IgE assay is also predictive of clinical reactivity during double-blind, placebo-controlled food challenge with cutoffs identified for egg, cow's milk, peanut and fish. The evidence is so strong that the use of these challenge tests in this setting raises ethical and medical responsibility issues. More recently, the intensity of sensitization, assessed by wheal diameter on skin tests and by specific IgE levels, has been shown to be a predictive factor for subsequent acquisition of immune tolerance in cow's milk protein allergy [4].

The development of recombinant allergens now makes possible a much more precise identification of the pollens responsible. For example, in tree pollen allergy, and in patients with positive skin tests for birch and ash, the

\section{KARGER}

Fax +4161306 1234 E-Mail karger@karger.ch www.karger.com
(C) 2011 S. Karger AG, Basel

1018-2438/11/1564-0349\$38.00/0

Accessible online at:

www.karger.com/iaa
Correspondence to: Dr. Claude André

Scientific and Medical Department, Stallergènes SA

6 Rue Alexis de Tocqueville

FR-92183 Antony (France)

Tel. +33 1555920 87, E-Mail candre@ @tallergenes.fr 
presence of anti-Fra e $1 \mathrm{IgE}$ can be used to distinguish between real sensitization to ash and cross-sensitization to birch and grass pollens [5]. A molecular basis has therefore been identified to clarify certain doubtful cases. The combination of rye pollen and grass pollens, commonly performed in clinical practice, is not justified, as it has been known for many years that grass pollens alone are sufficient for diagnosis and treatment [6] and, in children, skin tests are positive for rye only when they are also positive for grass pollens [7]. This homology is now well described [8]. This progress should also clarify the ambiguity constituting the basis for a combination of plantain immunotherapy and grass pollen immunotherapy in a difficult clinical context as the two plants flower at the same time. In a large number of cases, the positive skin test to plantain corresponds to cross-reactivity with grass group 5 allergens [9], a situation in which demonstration of specific anti-Pla $11 \mathrm{IgE}$ could be particularly useful [10]. Mugwort pollen allergy might also be overdiagnosed since positive test results may evolve due to cross-reactivity to other weeds [11].

Peanut constitutes a precedent for the prediction of the severity of clinical symptoms [12], as individual assessment of sensitization to peanut major allergens can distinguish between simple asymptomatic sensitization and clinical allergy. This study based on a cohort of 200 children sensitized to peanut or birch pollen, assessed at the age of 8 years, was predictive of the severity of clinical symptoms. Children sensitized to Ara h 2 responded with symptoms after contact with peanut in $83 \%$ of cases, while only $18 \%$ of children with isolated sensitization to Ara h 8 experienced symptoms and, most importantly, these symptoms were considerably milder. Monosensitization to Ara h 8 was almost only observed in subjects with intense sensitization to Bet $\mathrm{v} 1$. A possible explanation for this finding would be the resistance to heat and digestion of Ara h 1, Ara h 2 and Ara h 3 in contrast with the sensitivity to heat and digestion of Ara $h$ 8. Sensitization to Ara h 1 or Ara h 3 is observed only in the presence of sensitization to Ara h 2, resulting in reactivity to peanut in $95 \%$ of the cases, associated with more severe clinical reactions, with the presence of respiratory symptoms in $50 \%$ of the cases.

Pastorello et al. [1] reported remarkable results for peach major allergens by providing qualitative confirmation of the results obtained with individual peanut allergens [12], but especially quantitative conclusions concerning the risk level. This study was also conducted prospectively, ensuring more reliable definition and scoring of the severity of symptoms. One hundred and forty-eight patients, living in the same region, were classified into 4 grades of peach oral allergy syndrome (OAS): grade I (isolated OAS), grade II (OAS + gastrointestinal symptoms), grade III (OAS + systemic symptoms, urticaria, angioedema, rhinoconjunctivitis, asthma) and grade IV (OAS + life-threatening symptoms, glottis edema, hypotension, syncope). Grade I patients with so-called mild symptoms were compared to grade II + III + IV patients with so-called severe symptoms. As patients of this geographical region were also exposed to birch pollen, specific plasma IgE assays were performed for recombinant Pru p 1, Pru p 3, Pru p 4, Bet v 1, Bet v 2 and Bet v 4. One of the most important findings was that skin reactivity for peach lipid transfer protein (LTP) was positively correlated with anti-rPru p 3 IgE and negatively correlated with anti-rPru p 1 IgE. A significantly higher percentage of subjects with grade I OAS presented sensitization to birch, grasses, wall pellitory and ragweed, but not mugwort. Eighty-seven percent of patients with severe OAS were sensitized to Pru p 3, i.e. 6 times more often than grade I patients. An inverse proportion was observed for sensitization to birch. The anti-Pru p 1, anti-Pru p 4, anti-Bet $v 1$ and anti-Bet v 2 IgE concentrations were significantly higher in grade I patients, while the anti-Pru $\mathrm{p}$ $3 \mathrm{IgE}$ concentration was significantly higher in patients with severe OAS. A negative correlation was observed between anti-Pru $p 1$ IgE and anti-Pru p 3 IgE, but also with the severity of symptoms. A $1 \mathrm{kUA} / \mathrm{l}$ increase of anti-Pru p 1 corresponded to a $2.5 \%$ reduction in the odds of developing severe OAS, but the same $1 \mathrm{kUA} / \mathrm{l}$ increase of anti-Pru p $3 \mathrm{IgE}$ corresponded to a $9 \%$ increase in the odds of developing severe OAS. Analysis of sensitivity and specificity indicated that a value of $2.69 \mathrm{kUA} / \mathrm{l}$ of anti-rPru $\mathrm{p} 3$ discriminated peach-allergic patients at risk for developing more severe symptoms, but antipeach $\operatorname{IgE}$ values did not reach a similar positive cutoff. As Pru p 3 is an LTP resistant to gastric enzymes, it would be useful to verify the exact nature of the sensitization of the 19 patients with grade II OAS, as their gastrointestinal reactivity must be considered to be a local phenomenon and not a systemic reaction.

In this context, as local specific immunotherapy has been validated for the sublingual-swallow method but not for the sublingual-spit method, anti-LTP IgE assay could be useful to explain and prevent adverse effects of local specific immunotherapy. This predictive role needs to be verified case by case due to the limited cross-reactivity between mugwort Art v 3 and ragweed Amb a 6, explained by the low sequence identity $(32 \%)$ between these two LTPs [13]. 
Finally, as specific immunotherapy, regardless of its modality of administration, by injection or via the sublingual route, has been clearly shown to be effective only when a sufficient dose is administered, short-term and long-term therapeutic efficacy may only be achieved when each of the individual allergens responsible for clinical reactions is delivered at a sufficient dose, i.e. 5-20 $\mu \mathrm{g}$ of each allergen, as monthly maintenance treatment for injection immunotherapy and at an at least 10-fold higher daily dose for sublingual immunotherapy.

\section{References}

1 Pastorello EA, Farioli L, Pravettoni V, Scibilia J, Mascheri A, Borgonovo L, Piantanida M, Primavesi L, Stafylaraki C, Pasqualetti S, Schroeder J, Nichelatti M, Marocchi A: Pru p 3 sensitised Italian peach-allergic patients are less likely to develop severe symptoms when also presenting IgE antibodies to Pru p 1 and Pru p 4. Int Arch Allergy Immunol 2011;156:362-372.

2 Napoly A, Leveau C, Brunet D, Paupe J, Scheinmann P: Critères de mesure et seuil de positivité du prick-test à Dermatophagoides pteronyssinus chez l'enfant. Rev Fr Allergol 1992;32:180-186.

-3 Sampson HA, Ho DG: Relationship between food-specific IgE concentrations and the risk of positive food challenges in children and adolescents. J Allergy Clin Immunol 1997; 100:444-451.

4 Santos A, Dias A, Pinheiro JA: Predictive factors for the persistence of cow's milk allergy. Pediatr Allergy Immunol 2010;21: 1127-1134.
5 Niederberger V, Purohit A, Oster JP, Spitzauer S, Valenta R, Pauli G: The allergen profile of ash (Fraxinus excelsior) pollen: cross-reactivity with allergens from various plant species. Clin Exp Allergy 2002;32:933-941.

$\checkmark 6$ Lowenstein $\mathrm{H}$, Wihl JA, Billesbolle KB Bowadt H: Rationale for specific immunotherapy of grass pollen allergy with extracts of rye pollen. Allergy 1984;39:421-432.

7 Lelong M, Thibaudon M, Thelliez PH: Is it necessary to test children having summer respiratory problems with cereal pollens? Allerg Immunol (Paris) 1989;21:394-395

$\checkmark 8$ Hrabina M, Jain K, Gouyon B: Cross-reactivity between pollen allergens from common Pooideae grasses and cultivated cereals. Clin Exp Allergy Rev 2008;8:18-20.

$\checkmark$ Asero R, Mistrello G, Roncarolo D, Casarini M: Detection of allergens in plantain (Plantago lanceolata) pollen. Allergy 2000;55: 1059-1062.
10 Calabozo B, Diaz-Perales A, Salcedo G, Barber Domingo, Polo F: Cloning and expression of biologically active Plantago lanceolata pollen allergen Pla 11 in the yeast Pichia pastoris. Biochem J 2003;372:889-896.

1 Movérare R, Larsson H, Carlsson R, Holmquist I: Mugwort-sensitized individuals from North Europe, South Europe and North America show different IgE reactivity patterns. Int Arch Allergy Immunol 2011; 154:164-172.

12 Asarnoj A, Moverare R, Ostblom E, Poorafshar M, Lilja G, Hedlin G, van Hage M, Ahlstedt S, Wickman M: IgE to peanut allergen components: relation to peanut symptoms and pollen sensitization in 8-year-olds. Allergy 2010;65:1189-1195

13 Gadermaier G, Wopfner N, Wallner M, Egger M, Didierlaurent A, Regi G, Aberger F, Lang R, Ferreira F, Hawranek T: Array-based profiling of ragweed and mugwort pollen allergens. Allergy 2008;63:1543-1549. 